\title{
Exposition au rayonnement cosmique : surveillance du personnel de l'Armée de l'air navigant sur E-3F
}

\author{
J.-C. AMABILE ${ }^{1}$, X. CASTAGNET ${ }^{1}$, H. DE CARBONNIÈRES ${ }^{1}$, P. LAROCHE$^{1}$
}

(Manuscrit reçu le 22 juin 2006, accepté le 21 décembre 2006)

RÉSUMÉ Cette étude de poste repose, dans un premier temps, sur l'analyse d'une expérimentation consacrée aux mesures des débits d'équivalent de dose ambiante à bord des aéronefs de type E-3F. Ces résultats partiels sont complétés dans un second temps par une campagne de mesures de l'exposition individuelle à l'aide de dosimètres passifs et opérationnels gamma et neutrons. Les équipages de l'Armée de l'air navigant à bord des E-3F sont susceptibles de recevoir annuellement, à partir du niveau 300 et de 200 heures de vol, une dose efficace supérieure à $1 \mathrm{mSv}$. Il est donc impératif d'organiser une surveillance radiologique et médicale spécifique des équipages correspondant à ces critères de vol.

ABSTRACT Cosmic radiation exposure: work-place study about air-crew in the French Air-force flying on board the E-3F.

This workplace study is allowed to cosmic radiation of aircraft-crews. In a first time, the work is based on measurements of the ambient dose equivalent rates on board the E-3F aircraft. These results are supplemented by measurements of individual exposure using passive and active gamma and neutrons dosemeters. Above 10000 meters and 200 hours per year, the aircraft-crews flying on board the E-3F aircraft are likely to annually receive an effective dose higher than $1 \mathrm{mSv}$. It is therefore necessary to organize a specific radiological and medical survey of the aircraft-crews corresponding to these flight criteria.

Keywords: aviation / cosmic radiation / dosimetry / radiation protection

\section{Introduction}

La réglementation spécifique relative à la radioprotection a été récemment renforcée par la transposition en droit français de la directive 96/29/EURATOM (1996). Le code de la santé publique et le code du travail reprennent désormais les trois grands principes de la radioprotection énoncés par la Commission internationale de protection radiologique (CIPR) : la justification des pratiques, l'optimisation de la protection et la limitation des doses individuelles. La prévention du risque d'exposition aux rayonnements ionisants est basée sur le

${ }^{1}$ Service de protection radiologique des armées (SPRA), 1bis rue du Lieutenant Raoul Batany, 92141 Clamart Cedex, France. 
respect des trois principes de la radioprotection mais aussi, à l'instar des autres risques ou nuisances, sur l'évaluation préalable du risque ainsi que sur l'observation détaillée du poste de travail.

Dans le domaine du transport aérien, l'arrêté du 8 décembre 2003 fixe les modalités de mise en œuvre de la protection contre les rayonnements ionisants des travailleurs affectés à l'exécution de tâches à bord d'aéronefs en vol. Il impose désormais au chef d'établissement de mesurer l'exposition du personnel au rayonnement cosmique et d'en tirer les conséquences éventuelles en matière de surveillance médicale.

Le travail présenté est relatif à l'exposition au rayonnement cosmique des équipages de l'Armée de l'air navigant à bord des aéronefs de type E-3F (ou plus communément $A W A C S$ ). Leur mission principale est la surveillance et la gestion de l'espace aéro-maritime par des vols souvent de longue durée (plusieurs heures) à une altitude opérationnelle de 29000 pieds, soit légèrement inférieure à 10000 mètres.

L'objectif est de déterminer si les équipages de l'Armée de l'air navigant à bord de ces aéronefs sont susceptibles de recevoir annuellement une dose efficace supérieure à $1 \mathrm{mSv}$ (limite du public), en vue de mettre en œuvre des moyens de prévention adaptés et de déterminer les conditions dans lesquelles doivent être effectuées la surveillance radiologique et la surveillance médicale.

Dans un premier temps, a été réalisée une campagne de mesure des débits de dose ambiante à bord des E-3F, d'octobre 2000 à juin 2001. Ces résultats partiels ont été complétés dans un second temps par la mesure des expositions individuelles à l'aide de dosimètres passifs et opérationnels gamma et neutrons, entre septembre 2004 et février 2005.

\section{Rappel sur le rayonnement cosmique}

L'Homme vit dans un milieu où les rayonnements ionisants sont naturellement présents. L'exposition naturelle qui en résulte a pour origine les rayonnements cosmiques et telluriques, responsables d'expositions externes et internes. À cette irradiation naturelle s'ajoute l'irradiation liée aux activités humaines, notamment médicales.

L'UNSCEAR (United Nations Scientific Committee on the Effects of Atomic Radiation) évalue à $2,4 \mathrm{mSv}$ la dose efficace moyenne annuelle mondiale par habitant liée à la radioactivité naturelle (UNSCEAR, 2000). 
Le rayonnement cosmique primaire a deux origines (Planel, 2003) :

- le rayonnement galactique, provenant de l'explosion d'étoiles ou supernovae, qui est composé de protons ( $87 \%$ ), d'électrons, mais également de neutrons et d'ions lourds ;

- le vent solaire, composé de protons (99\%) et d'électrons, dévié par le champ magnétique terrestre et qui concentre ses lignes de force vers les régions polaires. Les éruptions solaires sont imprévisibles et leur influence à basse altitude n'est sensible que pour des éruptions majeures extrêmement rares. La dose reçue par les passagers d'un vol à 10000 mètres et à grande latitude effectué pendant l'éruption solaire de 1956, la plus importante connue aujourd'hui, est estimée à $10 \mathrm{mSv}$ (Reitz, 1993).

L'activité solaire suit en moyenne un cycle de onze ans. Lors des périodes d'activité solaire maximale, le champ magnétique d'origine solaire s'accroît, ce qui constitue un obstacle au cheminement des particules du rayonnement galactique (ce phénomène s'inverse quand l'activité solaire diminue).

Du fait de la nature dipolaire du champ magnétique terrestre et de sa rigidité magnétique, la pénétration des particules est plus importante au niveau des pôles que de l'équateur, où l'exposition sera donc plus faible.

Aux niveaux où s'effectuent les vols subsoniques et supersoniques les particules cosmiques, qu'elles soient d'origine solaire ou galactique, ont déjà traversé les hautes couches de l'atmosphère terrestre. Ces interactions avec les noyaux présents dans l'atmosphère $(\mathrm{H}, \mathrm{O}, \mathrm{N}$ et $\mathrm{C}$ principalement) produisent des particules chargées secondaires diverses dont l'énergie est plus faible que celle des particules incidentes. Ces interactions dépendent de l'énergie initiale des particules et des coordonnées géomagnétiques.

Aux altitudes habituelles de vol, l'exposition des personnels navigants est surtout due aux particules secondaires provenant de toutes ces interactions : pour moitié environ des neutrons, l'autre moitié étant constituée essentiellement d'électrons et photons.

Au total, l'exposition externe due au rayonnement cosmique est estimée, au niveau de la mer, à $0,4 \mathrm{mSv} / \mathrm{an}$. Elle varie en fonction de la latitude (elle est plus forte aux pôles qu'à l'équateur), de l'altitude (sa valeur double environ tous les $1500 \mathrm{~m}$ ) mais aussi en cas d'éruption solaire.

\section{Matériel et méthode}

Cette étude de poste repose la dosimétrie gamma et neutrons :

- dosimétrie d'ambiance d'une part,

- dosimétrie individuelle, active et passive, d'autre part. 


\subsection{Présentation de l'expérimentation consacrée aux mesures de débit de dose ambiante}

Afin de répondre au besoin exprimé par une expérimentation adaptée, l'examen des différents critères influençant le niveau de l'exposition a été réalisé en examinant les conditions de vol au sein de l'Armée de l'air (SPRA, 2002).

\subsubsection{Influence des éruptions solaires}

La période 2000-2001 correspondait à un maximum de l'activité solaire, donc à une diminution du niveau de rayonnements. Lors des minima du cycle solaire, il est possible d'atteindre des niveaux d'exposition supérieurs de $20 \%$ environ. Les manifestations aléatoires des éruptions solaires, rendent illusoire la possibilité d'anticiper leur importance, y compris par des mesures répétées. L'hypothèse de multiplier les expérimentations pour tenter d'observer l'émergence d'une éventuelle protubérance n'a donc pas été retenue.

\subsubsection{Choix des latitudes à explorer}

Les missions des aéronefs de l'Armée de l'air ont été étudiées en fonction de leur durée, des zones géographiques concernées et des altitudes d'action des différents appareils. Les appareils de transport, de reconnaissance, de surveillance et les avions d'arme présentaient des durées de vol très variables sous des latitudes différentes (France, Kosovo, Afrique entre tropique nord et équateur et pôle nord). Cette dernière latitude était intéressante par sa position géomagnétique mais la durée des vols était inférieure à dix heures par an (entraînements des escadrilles de reconnaissance aérienne): une étude spécifique pourrait être envisagée dans l'avenir. La latitude de la France, qui représentait $95 \%$ des missions de l'Armée de l'air, a donc été choisie pour cette expérimentation.

\subsubsection{Choix des aéronefs à contrôler}

Les différents types d'avions à expertiser ont été passés en revue en fonction de deux critères, les durées et les altitudes de vol: il en ressortait que l'avion effectuant les vols les plus longs aux altitudes les plus élevées est l'E-3F.

Le fait de disposer d'un aéronef spécialement pour l'expérience permettait d'effectuer des vols en palier et de multiplier les mesures pour chaque palier. C'est ce qui a été réalisé au cours de quatre vols d'essai d'octobre 2000 à juin 2001.

\subsubsection{Choix des moyens de détection}

Ces mesures, afin de ne pas subir de perturbations électromagnétiques, ont été réalisées en l'absence de fonctionnement des systèmes de détection de l'aéronef. 
Les appareils suivants ont été retenus pour cette expérimentation :

- débitmètre gamma ultra sensible. C'est un compteur proportionnel hélium méthane FH $40 \mathrm{G} 10$, permettant des mesures à partir de $30 \mathrm{nSv} / \mathrm{h}$ pour des photons de $30 \mathrm{keV}$ à $3 \mathrm{MeV}$. Son étalonnage est réalisé avec du cobalt-60. Pour les énergies supérieures, la mise à l'équilibre électronique est insuffisante et le détecteur minimise le résultat ;

- débitmètre neutron ultra sensible. C'est un rem compteur avec compteur hélium et modérateur de polyéthylène, permettant des mesures à partir de $10 \mathrm{nSv} / \mathrm{h}$ pour des neutrons depuis l'énergie thermique jusqu'à $20 \mathrm{MeV}$. L'étalonnage a été réalisé avec des champs réalistes (américium-beryllium ou californium). Pour des énergies supérieures, la réponse du compteur sous estime la dose réelle ;

- compteur proportionnel équivalent tissu (CPET) NAUSICAA. Ce type de compteur proportionnel à dépôt d'énergie est l'instrument de référence pour la détermination de l'équivalent de dose ambiante $\mathrm{H}^{*}(10)$. Il est rempli d'un mélange de gaz argon-méthane équivalent tissu sous faible pression. Il a été étalonné dans les champs mixtes américium-beryllium et cobalt-60. Des mesures effectuées auprès des installations du CERN, à des énergies variées de particules ont montré que les étalonnages de ces compteurs effectués à plus basses énergies restent valables pour les types de particules trouvées en altitude.

\subsection{Présentation de la campagne de mesures de l'exposition individuelle}

Cette partie de l'étude a pour objectif de déterminer si les équipages navigants à bord des E-3F sont susceptibles de recevoir annuellement une dose efficace supérieure à $1 \mathrm{mSv}$.

Les résultats des mesures des débits de dose ambiante à bord des E-3F seront complétés par l'évaluation de l'exposition individuelle de six personnels navigants pouvant voler sur trois appareils de ce type. Les dosimètres passifs et opérationnels, gamma et neutrons, seront portés plus de quatre mois, du 30 septembre 2004 au 17 février 2005.

\subsubsection{Moyens de surveillance dosimétrique}

\section{- Dosimétrie passive bêta, $\mathrm{X}$ et gamma : dosimètre $\mathrm{OSL}$}

Au sein du ministère de la défense, le dosimètre photoluminescent de technologie OSL (Optically Stimulated Luminescence) a remplacé le film photographique, pour une détection plus précise des rayonnements photoniques et bêta. Le capteur 
est composé d'une mince feuille de poudre d'oxyde d'aluminium dopé au carbone. La technologie OSL repose sur le principe de lecture d'une émission de lumière. Cette luminescence est provoquée par un flash lumineux (par laser ou diode électroluminescente). Lors d'une interaction avec un rayonnement ionisant, des charges se retrouvent piégées dans le matériau sensible. Ces électrons sont retenus dans ces pièges jusqu'à ce qu'ils reçoivent une excitation extérieure qui les libère. Ils se désexcitent alors par émission de luminescence dont l'énergie est proportionnelle à l'énergie initialement déposée par l'irradiation.

Le matériau est capable de mesurer des doses allant de $0,05 \mathrm{mSv}$ à $10 \mathrm{~Sv}$ et concerne les rayonnements gamma de $5 \mathrm{keV}$ à $40 \mathrm{MeV}$ et les bêta supérieurs à $150 \mathrm{keV}$. Il est insensible aux rayonnements neutroniques. Par rapport au film photographique, le seuil de détection est abaissé de $50 \%$ (Archambault et al., 2005).

\section{- Dosimétrie passive neutron : dosimètre NP3}

Le dosimètre NP3 (Neutron Plastique de $3^{\mathrm{e}}$ génération) permet de prendre en compte les neutrons entre $0,2 \mathrm{MeV}$ et $66 \mathrm{MeV}$, avec un seuil de détection à $0,2 \mathrm{mSv}$ (Barlow et al., 1997). Cette technologie permet d'identifier les traces de recul des protons produits par la diffusion élastique des neutrons rapides dans le matériau plastique CR39 (polycarbonate Colombia Resine 39). Les interactions des neutrons lents incidents sur un radiateur produisent, par capture, des particules chargées lourdes plus facilement détectables. L'étalonnage a été réalisé sur des spectres d'américium-béryllium (spectre dur), de californium (spectre de fission) ou de californium modéré $\mathrm{DO}_{2}$ (spectre dégradé).

L'étui accueillant le dosimètre OSL a été adapté pour pouvoir y loger le détecteur neutrons de type NP3. Le personnel porte ainsi ses deux détecteurs dans un boîtier unique. La lecture est automatique.

\section{- Dosimétrie active $X$ et gamma : le dosimètre DMC 2000S}

Ce dosimètre est un détecteur à semi-conducteurs en silicium conditionné dans un boîtier pourvu d'un afficheur à cristaux liquides. La détection des photons $\mathrm{X}$ et gamma est possible grâce à l'augmentation temporaire de la conductivité électrique des semi-conducteurs après ionisation. Ces dosimètres fonctionnent comme des chambres d'ionisation solides et produisent un courant dont l'intensité est proportionnelle à l'énergie cédée. Ils mesurent des équivalents de dose absorbée pour des rayonnements $\mathrm{X}$ ou gamma d'énergie comprise entre $60 \mathrm{keV}$ et $3 \mathrm{Mev}$, depuis $1 \mu \mathrm{Sv}$ jusqu'à $10 \mathrm{~Sv}$. Ils mesurent des débits d'équivalent de dose absorbée compris entre $10 \mu \mathrm{Sv} / \mathrm{h}$ et $1 \mathrm{~Sv} / \mathrm{h}$. Deux seuils d'alarme existent, l'un pour l'équivalent de dose absorbée, l'autre pour le débit d'équivalent de dose absorbée. 


\section{- Dosimétrie « active neutron » : dosimètre à bulles}

Le dosimètre à bulles se présente sous la forme d'un stylo renfermant quelques $\mathrm{cm}^{3}$ d'un gel polymère transparent équivalent tissu contenant des microgouttelettes de fréon. En période d'utilisation, le gel est décomprimé et le fréon, mis en sur-ébullition, devient alors instable. Lors de l'irradiation l'énergie libérée dans le gel par des particules à transfert linéique d'énergie élevé, notamment des neutrons, provoque une vaporisation des micro-gouttelettes jusqu'à une taille où elles deviennent visibles et peuvent être facilement comptées à l'œil (ou par un système optique). Le nombre de bulles créé est proportionnel à celui des collisions, la sensibilité (en $\mu \mathrm{Sv} / \mathrm{bulle}$ ) est indiquée sur le dosimètre. Ce dosimètre permet ainsi, par son information visuelle, de sensibiliser les travailleurs à leur exposition.

En période de stockage, le dosimètre est rendu insensible aux rayonnements par maintien sous pression. Le dosimètre à bulles présente néanmoins des inconvénients (coût, coupure en énergie à environ $200 \mathrm{keV}$, sensibilité aux chocs, à la température, durée de vie limitée), mais il permet d'évaluer l'équivalent de dose neutron à $50 \%$ (Rannou et al., 1998). Ces dosimètres doivent être maintenus à l'abri de toute source de rayonnements, de chaleur et d'humidité.

Pour la surveillance dosimétrique active (ou opérationnelle), dix dosimètres neutrons individuels à bulles et dix dosimètres individuels de type SOR (photons) sont distribués aux six membres d'équipage suivis. Quatre dosimètres de chaque type sont gardés en «secours » et aucun n'est utilisé en tant que dosimètre d'ambiance.

\subsubsection{Protocole}

La surveillance dosimétrique individuelle a été réalisée pendant six mois sur six personnels navigant sur les E3-F. Chacun d'entre eux portait :

- une pochettes contenant les dosimètres passifs gamma OSL et neutron NP3,

- un dosimètre actif $X$ et gamma DMC 2000S,

- un dosimètre « actif » neutron à bulles.

Les ambiances des trois aéronefs ont été également surveillées pendant toute la durée de l'étude, à raison de deux pochettes « ambiance » (OSL et NP3 par avion ; une pochette à proximité du radar, la seconde au niveau du lobe arrière). Enfin une dosimétrie témoin a été mise en place à terre sur le poste de repos des dosimètres individuels non utilisés.

Des instructions ont été données au personnel impliqué dans cette étude de poste afin d'obtenir des résultats précis et fiables. Elles concernaient notamment :

- l'identification des dosimètres individuels passifs (en notant le nom du porteur sur la pochette) ; 
- les précautions d'emploi des dosimètres à bulles : remise en compression (pour une durée de douze heures) une fois par semaine d'utilisation ou dès que l'on peut compter plus de dix bulles (en revanche, il ne faut jamais comptabiliser les grosses bulles ou les paquets de bulles généralement dus à un choc);

- les informations fournies sur le bordereau des dosimètres : nom, prénom, date de naissance et la case «observations» renseignée en cas de choc du dosimètre, d'avarie ou de remarques diverses ;

- la localisation du poste de repos pour les dosimètres non utilisés et le dosimètre témoin ;

- l'installation de la dosimétrie d'ambiance sur l'E-3F, dans les endroits repérés au préalable, pour toute la durée de l'étude ;

- l'intérêt du cahier de dosimétrie. Ce cahier de dosimétrie est rempli à la fin de chaque vol et émargé par le chef de bord. Il permet de noter des renseignements précieux concernant l'utilisation:

- des pochettes individuelles contenant les dosimètres passifs : nom du porteur, numéro de la pochette, numéro de la mission et date, paramètres de vol (temps de vol, altitudes caractéristiques, observations éventuelles, ...);

- des dosimètres neutrons à bulles (nombre de bulles avant et après le vol) ;

- des dosimètres actifs DMC 2000S (lecture directe).

\section{Résultats}

\subsection{Résultats des mesures de débit de dose ambiante}

Au total la campagne consacrée à l'étude de la variation du débits d'équivalent de dose ambiante en fonction de l'altitude permet de retenir des niveaux de l'ordre de :

- 4 à 5,5 $\mu \mathrm{Sv}$ par heure au niveau 290 (29 000 pieds soit environ 9600 mètres d'altitude),

- 6 à $8 \mu \mathrm{Sv}$ par heure au niveau 400 (40 000 pieds soit environ 13300 mètres d'altitude).

En dessous du niveau 290, les valeurs des débits de dose ambiante présentent de trop nombreuses fluctuations erratiques.

\subsection{Résultats des mesures de l'exposition individuelle}

\subsubsection{Dosimétrie d'ambiance}

Les résultats de la dosimétrie passive d'ambiance sont présentés dans le tableau I. Les dosimètres placés dans le lobe arrière sont situés à proximité du système radar. La limite de détection du NP3 n'est pas suffisante pour dans conditions de mesures. 
TABLEAU I

Équivalent de dose ambiante $\left(\mathrm{H}^{*} 10\right)$ en dosimétrie passive. Ambiant equivalent dose $\left(\mathrm{H}^{*} 10\right)$ with passive dosimeters.

\begin{tabular}{llccc}
\hline $\begin{array}{l}\text { Période maximale d'étude : } \\
\text { quatre mois et demi }\end{array}$ & $\begin{array}{c}\text { Dose NP3 } \\
(\mathrm{mSv})\end{array}$ & $\begin{array}{c}\text { Dose OSL } \\
(\mathrm{mSv})\end{array}$ & $\begin{array}{c}\text { Dose totale } \\
\text { en dosimétrie passive } \\
\text { d'ambiance }(\mathrm{mSv})\end{array}$ \\
\hline Ambiance E-3F A : & lobe arrière & 0 & 0,15 & 0,15 \\
\hline Ambiance E-3F A : & consoles & 0 & 0 & 0 \\
\hline Ambiance E-3F B : & lobe arrière & 0 & 0,45 & 0,45 \\
\hline Ambiance E-3F B : & consoles & 0 & 0,25 & 0,25 \\
\hline Ambiance E-3F C : & lobe arrière & 0 & 0,15 & 0,15 \\
\hline Ambiance E-3F C : & consoles & 0 & 0,10 & 0,10 \\
\hline
\end{tabular}

\section{TABLEAU II}

Équivalents de dose $\mathrm{Hp}(10)$ par dosimétrie individuelle gamma et neutron. Individual equivalent dose $\mathrm{Hp}(\mathbf{1 0})$ gamma and neutron.

\begin{tabular}{lccccccc}
\hline $\begin{array}{l}\text { Niveau } \\
\text { moyen } \\
\text { d'altitude }(*)\end{array}$ & $\begin{array}{c}\text { Durée } \\
\text { du vol } \\
(\mathrm{min})\end{array}$ & $\begin{array}{c}\text { Dosimétrie } \\
\text { active } \\
(\mu \mathrm{Sv})\end{array}$ & $\begin{array}{c}\text { Débit de } \\
\text { dose } \\
(\mu \mathrm{Sv} / \mathrm{h})\end{array}$ & $\begin{array}{c}\text { Temps pour } \\
\text { atteindre } \\
1 \mathrm{mSv} / \mathrm{an}(\text { heures })\end{array}$ & $\begin{array}{c}\text { Dosimétrie } \\
\text { passive } \\
(\mu \mathrm{Sv})\end{array}$ & $\begin{array}{c}\text { Débit de } \\
\text { dose } \\
(\mu \mathrm{Sv} / \mathrm{h})\end{array}$ & $\begin{array}{c}\text { Temps pour } \\
\text { atteindre } \\
\mathrm{mSv} / \text { an (heures })\end{array}$ \\
\hline 302 & 3000 & 205 & 4,1 & 244 & 0,10 & 2 & 500 \\
\hline 294 & 7540 & 234 & 1,86 & 538 & 0,20 & 1,59 & 629 \\
\hline 291 & 4950 & 233 & 2,82 & 355 & & & \\
303 & 4800 & 246 & 3,08 & 325 & & & \\
\hline 300 & 3130 & 127 & 2,43 & 412 & & & 208 \\
\hline 304 & 2490 & 195 & 4,70 & 213 & 0,20 & 4,82 & \\
\hline
\end{tabular}

*) Pour obtenir l'altitude en mètres, multiplier le niveau moyen d'altitude par 100, puis diviser par trois la mesure obtenue. Le niveau 300 correspond à 30000 pieds soit environ 10000 mètres.

\subsubsection{Dosimétrie individuelle}

Les résultats de la dosimétrie active et passive des six personnels navigants suivis au cours de cette étude de poste sont détaillés, en fonction des durées de vol et des niveaux d'altitude moyens, dans le tableau II. Le nombre d'heures pour atteindre la limite annuelle de $1 \mathrm{mSv}$ est précisé pour chaque individu.

\subsubsection{Dosimétrie opérationnelle}

La dosimétrie active a permis de déterminer des débits de dose en fonction des niveaux d'altitude moyens. Les résultats sont reportés dans le tableau III. 
TABLEAU III

Équivalent de dose $\mathrm{Hp}(10)$ et débits d'équivalent de dose en dosimétrie active en fonction des niveaux moyens d'altitude.

Variation of equivalent dose $\mathrm{Hp}(10)$ with altitude, obtained with active dosimeters (dose and dose rate).

\begin{tabular}{lccccccc}
\hline $\begin{array}{l}\text { Durée } \\
\text { de vol } \\
(\text { min })\end{array}$ & $\begin{array}{c}\text { Altitudes } \\
\text { moyennes } \\
(\text { niveaux })\end{array}$ & $\begin{array}{c}\text { Dose } \\
\text { neutron } \\
(\mu \mathbf{S v})\end{array}$ & $\begin{array}{c}\text { Dose } \\
\text { SOR } \\
(\mu \mathbf{S v})\end{array}$ & $\begin{array}{c}\text { Dose } \\
\text { totale } \\
(\mu \mathbf{S v})\end{array}$ & $\begin{array}{c}\text { Débit de dose } \\
\text { neutron } \\
(\mu \mathbf{S v} / \mathbf{h})\end{array}$ & $\begin{array}{c}\text { Débit de } \\
\text { dose SOR } \\
(\mu \mathbf{S v} / \mathbf{h})\end{array}$ & $\begin{array}{c}\text { Débit de dose } \\
\text { total } \\
(\mu \mathbf{S v} / \mathbf{h})\end{array}$ \\
\hline 140 & 100 & 4 & 0 & 4 & 1,71 & 0 & 1,71 \\
\hline 40 & 150 & 0 & 0 & 0 & 0,00 & 0 & 0,00 \\
\hline 170 & 190 & 13 & 0 & 13 & 4,59 & 0 & 4,59 \\
\hline 490 & 200 & 18 & 10 & 28 & 2,20 & 1,22 & 3,43 \\
\hline 140 & 230 & 0 & 0 & 0 & 0,00 & 0,00 & 0,00 \\
\hline 450 & 240 & 0 & 0 & 0 & 0,00 & 0,00 & 0,00 \\
\hline 160 & 250 & 0 & 10 & 10 & 0,00 & 3,75 & 3,75 \\
\hline 100 & 265 & 0 & 0 & 0 & 0,00 & 0,00 & 0,00 \\
\hline 390 & 285 & 4 & 0 & 4 & 0,62 & 0,00 & 0,62 \\
\hline 4570 & 290 & 192 & 90 & 282 & 2,52 & 1,18 & 3,70 \\
\hline 7490 & 300 & 251 & 100 & 351 & 2,01 & 0,80 & 2,81 \\
\hline 10280 & 310 & 326 & 140 & 466 & 1,90 & 0,82 & 2,72 \\
\hline 490 & 320 & 13 & 10 & 23 & 1,59 & 1,22 & 2,82 \\
\hline 1000 & 330 & 39 & 20 & 59 & 2,34 & 1,20 & 3,54 \\
\hline $\mathbf{2 5 9 1 0}$ & & & & & & & \\
\hline
\end{tabular}

Ces résultats de dosimétrie opérationnelle, relevés au total sur 431 heures et 50 minutes (25 910 minutes), aboutissent au graphique du débit de dose total en fonction de l'altitude moyenne. Ils sont reportés dans la figure 1.

\section{Analyse et discussion}

L'incertitude globale liée à la détermination de l'équivalent de dose par un dosimètre ne doit pas être négligée. Elle provient d'une part de l'incertitude aléatoire liée à la lecture du dosimètre et d'autre part à de nombreuses incertitudes systématiques qui affectent la réponse du dosimètre : étalonnage, dépendance énergétique et angulaire, non linéarité de la réponse, ...

L'incertitude pour les mesures de la composante photonique est de l'ordre de $\pm 15 \%$ pour les mesures de l'ordre du $\mu \mathrm{Sv} / \mathrm{h}$ (donnée constructeur). 


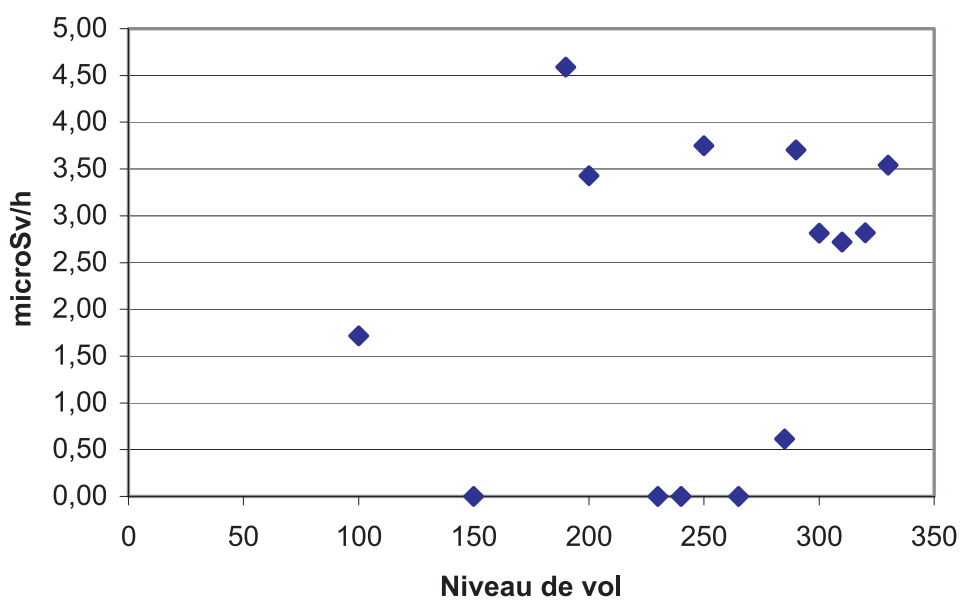

Figure 1 - Débits d'équivalent de dose ambiante $(H * 10)$ obtenus avec les dosimètres opérationnels en fonction des niveaux de vol moyens.

Ambiant equivalent dose rate obtained with active dosimeters as function of averaged flight level.

L'incertitude pour les mesures neutroniques varie de $\pm 10 \%$ pour les valeurs de l'ordre du $0,2 \mu \mathrm{Sv} / \mathrm{h}$ à $\pm 3,5 \%$ quand le débit d'équivalent de dose atteint $2 \mu \mathrm{Sv} / \mathrm{h}$.

Pour le compteur proportionnel équivalent tissu à dépôt d'énergie, la dispersion des mesures dépend de la statistique de comptage. De l'altitude zéro à l'altitude 400, celle-ci a varié de 0,2 impulsion par minute à 8 impulsions par minute. Ces valeurs faibles de la statistique, spécialement pour les faibles valeurs de l'équivalent de dose expliquent les variations enregistrées pour les mesures à une même altitude.

Par ailleurs les deux temps de cette étude de poste couvrent une période d'activité solaire maximale en 2000-2001, et une période d'activité quasi minimale en 2005, qui pourrait être à l'origine d'une majoration des doses reçues de $20 \%$.

Les mesures des débits de dose ambiante à bord des E-3F, comprises entre 4 et $8 \mu \mathrm{Sv}$ par heure pour des niveaux de 290 à 400, sont en accord avec les études réalisées sur les vols commerciaux (Bottollier-Depois et al., 2003). En haute altitude, la couche d'atmosphère protectrice se fait plus mince et l'exposition au rayonnement cosmique est plus importante. 
Le tableau I met en évidence le fait que la dosimétrie passive d'ambiance sur E-3F donne des résultats supérieurs au niveau du lobe arrière, c'est-à-dire à proximité du radar. La mesure de l'exposition dépendant peu de l'emplacement du dosimètre dans l'appareil, il n'est pas possible (compte tenu du faible nombre de relevés dosimétriques) d'identifier une quelconque interaction entre le rayonnement radar et les résultats de la dosimétrie.

La surveillance dosimétrique individuelle (Tab. II) nous montre que, dans certaines circonstances, il faudrait entre 200 et 250 heures de vol $(208-213-$ 244 heures) pour atteindre la limite annuelle de $1 \mathrm{mSv}$, pour une altitude moyenne située autour du niveau 300.

Les seuils de détection des dosimètres passifs sont supérieurs à ceux des dosimètres opérationnels, ce qui explique les résultats généralement inférieurs obtenus. L'absence de dose ne signifie pas l'absence d'une exposition. De même une faible dose détectée en dosimétrie active ne signifie pas une exposition plus importante.

D'autre part, des doses NP3 systématiquement notées à $0 \mathrm{mSv}$ traduisent des doses inférieures au seuil de détection et donc non significatifs au plan sanitaire.

En ce qui concerne la dosimétrie opérationnelle, le tableau III suggère que la composante neutronique relevée équivaudrait à environ $70 \%$ du rayonnement cosmique total (au lieu de $50 \%$ ). En revanche, le graphique des débits de dose en fonction de l'altitude moyenne (Fig. 1) est cohérent par rapport à l'augmentation de l'exposition au rayonnement cosmique avec l'altitude.

Les deux temps de cette étude de poste couvrent une période d'activité solaire maximale en 2000-2001 et une période d'activité quasi minimale en 2005 (cycle de onze ans), qui pourrait être à l'origine d'une majoration des doses reçues de $20 \%$. Il est important de noter que dans les deux cas, les détecteurs utilisés (débitmètres gamma et neutron ultra sensibles, Sievertmètre NAUSICAA ou dosimètres passifs OSL/NP3 et dosimètres actifs) mettent en évidence que les personnels navigant sur E-3F sont susceptibles de cumuler une dose efficace annuelle légèrement supérieure à $1 \mathrm{mSv}$ :

- au-delà de 200 heures de vol par an ;

- pour des niveaux d'altitude supérieur ou égal à 300 .

Ces personnels volent en moyenne 250 à 400 heures par an en temps de paix.

Dans le cadre d'une mission extérieure ou d'un conflit, ce temps de vol pourrait être très largement majoré. Un scénario enveloppe prévoyant 1000 heures de vol annuel, à $8 \mu \mathrm{Sv}$ par heure au niveau 400, aboutirait à une dose efficace annuelle de $8 \mathrm{mSv}$ au maximum. 


\section{Conclusion}

Dans le cadre de ses attributions, le Service de protection radiologique des armées (SPRA) participe à l'application, au sein des organismes du ministère de la défense, des mesures de protection des personnes contre les rayonnements ionisants (Arrêté du 10 janvier, 2005). À la suite de cette étude de poste, réalisée sur deux périodes, la totalité des recommandations formulées auprès du centre d'expériences aériennes militaires (CEAM) et à l'état major de l'armée de l'air (EMAA) a été adoptée.

L'ensemble des mesures fait apparaître, à partir du niveau 300 et de 200 heures de vol par an, un risque de dépassement de la limite « public » $(1 \mathrm{mSv})$. Il est donc impératif, dans ces conditions, d'organiser une surveillance radiologique et médicale.

Ainsi, les personnels navigants concernés doivent :

- être classés en catégorie $B$ par le commandement après avis du médecin de prévention ;

- recevoir une information initiale puis triennale sur la nature de l'exposition, les risques et les mesures de prévention mises en œuvre. À cet effet le logiciel SIEVERT [Système d'Information et d'Évaluation par Vol de l'Exposition au Rayonnement cosmique dans les Transports aériens], qui permet d'obtenir une évaluation de la dose reçue (Bottollier-Depois et al., 2003) par rayonnement cosmique, peut être présenté ;

- être sensibilisés, pour les personnels féminins, à la nécessité de déclarer précocement une grossesse. L'adaptation du poste de travail n'est pas envisagé du fait de l'inaptitude temporaire au service aérien jusqu' au terme de la grossesse ;

- recevoir, conformément à la réglementation de droit commun, une carte individuelle de suivi médical (catégorie B) délivrée par l'Institut de radioprotection et de sûreté nucléaire (IRSN) ;

- faire l'objet d'une surveillance médicale renforcée (visite initiale puis au moins une fois par an) et d'une fiche d'exposition individuelle insérée dans un dossier médico-radiobiologique ;

- bénéficier, pour toute mission aérienne, d'une dosimétrie passive trimestrielle individuelle associée à une dosimétrie d'ambiance (mesure spécifique à la défense).

Compte tenu des niveaux de dose enregistrés, des variations des débits de dose en fonction de l'altitude, et des circonstances d'exposition du personnel, aucun zonage n'est proposé pour l'instant sur les aéronefs de l'Armée de l'air. En revanche, le risque de franchissement de la limite «public» pour certains personnels est à l'origine d'une surveillance médicale spécifique, en plus de la surveillance de l'ambiance. 
La catégorisation des personnels navigants pourra être revue en fonction des résultats de la surveillance généralisée et de la situation opérationnelle. Par exemple, dans le cadre du scénario enveloppe de temps de crise précédemment décrit, une catégorisation A s'imposerait sans pour autant modifier les modalités de la surveillance médico-radiobiologique du temps de paix.

Ces mesures concernent les équipages des E-3F et les autres aéronefs correspondant à ces critères de vol : C 135 F, FALCON 50 et 900, A 310, A 319, les A 340 et A $400 \mathrm{M}$ (en commande). Elles concerneront probablement aussi, dans un proche avenir, les aéronefs de la marine nationale.

Remerciements. Les auteurs remercient chaleureusement les personnels de la BA 702 d'Avord, les lieutenants Divry et Maupas pour leur participation active à cette étude de poste, ainsi que Madame Briand-Champlong, Monsieur Aranjo et le capitaine Barrois du Service de protection radiologique des armées, pour leur soutien technique et leurs précieux conseils.

\section{RÉFÉRENCES}

Archambault V., Le Roy G., Prugnaud B. (2005) Dosimétrie passive: introduction d'un nouveau dosimètre basé sur la technologie OSL, Radioprotection 40, 503-507.

Arrêté du 10 janvier (2005) relatif aux attributions du Service de protection radiologique des armées.

Barlow K., Jackson M., French A., Harvey J.-R. (1997) Application of neutron spectrometry in the DRPS neutron dosimetry service, Rad. Prot. Dosim. 70, 265-268.

Bottollier-Depois J.-F., Biau A., Blanchard P., Clairand I., Dessarps P., Lantos P., Saint-Lô D., Valero M. (2003) Assessing exposure to cosmic radiation aboard aircraft : the SIEVERT system, Radioprotection 38, 357-366.

Directive 96/29/EURATOM (1996) du Conseil en date du 13 mai 1996 fixant les normes de base relatives à la protection sanitaire de la population et des travailleurs contre les dangers résultant des rayonnements ionisants.

Planel H. (2003) Le rayonnement cosmique, Urgence pratique 59, 39-41.

Rannou A., Bartge J., Aubert B., Brégeon B., Champlong J., Colson P., Espagnan M., Herbaut Y., Thevenin J.C., Valero M. (1998) Etat de l'art des techniques de dosimétrie individuelle et analyse des besoins, Radioprotection 33, 405-433.

Reitz G. (1993) Radiation environment in the stratosphere, Rad. Prot. Dos. 48, 5-20.

SPRA (2002) Rapport n² 236 DEF/SPRA/D du 19 juillet 2002.

UNSCEAR (2000) Sources and effects of ionizing radiation, Report to the General Assembly, Vol. 1 - annexe B: Exposures from natural radiation sources. United Nations sales publication E.00.IX.3. United Nations, New-York. 